\title{
On temporal behaviour of solutions in Thermoelasticity of porous micropolar bodies
}

\author{
Marin Marin and Olivia Florea
}

\begin{abstract}
We consider a porous thermoelastic body, including voidage time derivative among the independent constitutive variables. For the initial boundary value problem of such materials, we analyze the temporal behaviour of the solutions. To this aim we use the Cesaro means for the components of energy and prove the asymptotic equipartition in mean of the kinetic and strain energies.
\end{abstract}

\section{Introduction}

The high temperatures that act on the materials involve on these, during the normal usage, at one moment, a heat flow. The thermal stress is determined by the temperature distribution induced by the heat flow.

The magnitude of the thermal stress can be affected by the pertinent material properties, as well as by the others variables which appear in the changes of the material properties. In this analysis must be taken into account all the failure possibilities.

These notions have an applicative character in different domains of activity, which treat the porous materials like the geological materials, especially the rocks and the soil, like the manufactured materials, especially the solid packed granular, the ceramics and the pressed powder. The first researchers who made investigations on the porous materials were Goodman and Cowin, [1],

Key Words: micropolar, voids, thermoelastic, Cesaro mean, asymptotic equipartition. 2010 Mathematics Subject Classification: Primary 65M50, 65M30; Secondary 65N50.

Received: April 2013

Revised: May 2013

Accepted: October 2013 
who presented the granular theory. The same study was researched by Cowin and Nunziato, [2], whose aim was to discover the mechanical behaviour of the porous solids when the matrix material is elastic and the interstices are voids of materials. To respect this idea they introduced an additional degree of freedom.

The theory of Cowin and Nunziato ([3]) can be applied to the non conductibility thermal materials. This study is based on the material achieve which the bulk density could be written like a product of two fields: the matrix material density field and the volume fraction field (see also, [4], [5], [6]). Iesan studied the theory of the thermoelastic materials with voids, [4], making a direct generalization of the linear elastic body, neglecting the changes in the volume fraction due to the internal dissipation in the material.

Chirita and Ciarletta used the method for the time-weighted surface power function. In [7] it was studied the asymptotic behaviour of the solutions for the periodic competition diffusion systems. The classical functions of Liapunov are modified through some piecewise continuous functions, obtaining sufficient conditions for the asymptotic stability of the solutions, [8].

An elegant study of the solutions temporal behaviour for the theromelastic bodies with microstretch was made in our paper, [10], and in the paper [9] is proved the uniform dissipation of the energy for the thermoelastic bodies with microstretch.

In the present study we extend the Cowin and Nunziato theory to cover the micropolar themoelastic material by adding into the set of constitutive variables the time derivative of the voidage to include the inelastic effects.

\section{Basic equations}

At time $t=0$ a body occupies a properly regular region, denoted by $B$, of the Euclidian three-dimensional space $R^{3}$. In order to admit the application of the divergence theorem, we consider that the boundary of the properly region, denoted by $\partial B$, is a sufficiently smooth surface. The closure of $B$ is denoted by $\bar{B}$. In this paper we will study the motion of the continuum to a fixed system of rectangular Cartesian axes $O x_{i},(i=1,2,3)$ and adopt Cartesian tensor notation. The italic indices will always assume the values $1,2,3$, whereas the Greek indices will range over the value 1,2 . The material time derivative is expressed with a superposed dot, and the partial derivatives with respect to the spatial coordinates are expressed with a comma. In this paper is used the Einstein summation on the repeated indices and is omitted the spatial argument and the time argument of a function, when is no likelihood of confusion.

The bulk density $\varrho$ could be written like a product of two fields: the matrix 
material density field $\gamma$ and the volume fraction field $\nu$ :

$$
\varrho_{0}=\gamma_{0} \nu_{0}
$$

where $\gamma_{0}$ and $\nu_{0}$ are spatially constants. The motion of the micropolar thermoelastic body with voids is described by the independent variables:

- $u_{i}(x, t), \varphi_{i}(x, t)$ - the displacement and microrotation fields from reference configuration;

- $\theta$ - the change in temperature from $T_{0}$, the absolute temperature of the reference configuration, i.e. $\theta(x, t)=T(x, t)-T_{0}$;

- $\sigma$ - the change in volume fraction measured from the reference configuration volume fraction $\nu_{0}$, i.e. $\sigma(x, t)=\nu(x, t)-\nu_{0}$.

The free energy function, in the case that the initial body is stress free, with a null intrinsic equilibrated body force and a null flux rate, within the linear theory, is:

$$
\begin{aligned}
& \Psi=\frac{1}{2} A_{i j m n} \varepsilon_{i j} \varepsilon_{m n}+B_{i j m n} \varepsilon_{i j} \gamma_{m n}+\frac{1}{2} C_{i j m n} \gamma_{i j} \gamma_{m n}+ \\
& +B_{i j} \sigma \varepsilon_{i j}+C_{i j} \sigma \gamma_{i j}+D_{i j k} \phi_{k} \varepsilon_{i j}+E_{i j k} \phi_{k} \gamma_{i j}- \\
& -\alpha_{i j} \theta \varepsilon_{i j}-\beta_{i j} \theta \gamma_{i j}-m \theta \sigma+d_{i} \sigma \phi_{i}+\gamma_{i} \theta \phi_{i}- \\
& -\frac{1}{2} a \theta^{2}+\frac{1}{2} \xi \sigma^{2}+\frac{1}{2} A_{i j} \phi_{i} \phi_{j}-\frac{1}{2} \omega \dot{\sigma}^{2} .
\end{aligned}
$$

As in [2], $f=-\omega \dot{\sigma}$ is the dissipation which takes into account of the inelastic behaviour of the voids. Also, $\omega$ is a positive constant. Taking into account the free energy function, using a common method, we can obtain the following constitutive equations:

$$
\begin{aligned}
& t_{i j}=C_{i j m n} \varepsilon_{m n}+B_{i j m n} \gamma_{m n}+B_{i j} \sigma+D_{i j k} \phi_{k}-\beta_{i j} \theta, \\
& m_{i j}=B_{m n i j} \varepsilon_{m n}+C_{i j m n} \gamma_{m n}+C_{i j} \sigma+E_{i j k} \phi_{k}-\alpha_{i j} \theta, \\
& h_{i}=D_{m n i} \varepsilon_{m n}+E_{m n i} \gamma_{m n}+d_{i} \sigma+A_{i j} \phi_{j}-\gamma_{i} \theta, \\
& g=-B_{i j} \varepsilon_{i j}-C_{i j} \gamma_{i j}-\xi \sigma-d_{i} \phi_{i}+m \theta, \\
& \varrho \eta=\alpha_{i j} \varepsilon_{i j}+\beta_{i j} \gamma_{i j}+m \sigma+\gamma_{i} \phi_{i}+a \theta, \\
& q_{i}=k_{i j} \theta_{, j},
\end{aligned}
$$

where $\varepsilon_{i j}, \gamma_{i j}$ and $\phi_{i}$ are the kinematic characteristics of the strain and we have the following geometric relations:

$$
\varepsilon_{i j}=u_{j, i}+\varepsilon_{j i k} \varphi_{k}, \gamma_{i j}=\varphi_{j, i}, \phi_{i}=\sigma, i, \theta=T-T_{0}, \sigma=\nu-\nu_{0} .
$$

Taking into account the method use by Nunziato and Cowin in [3], the following fundamental equations are derived (se also, [9]): - the equations of 
motion:

$$
\begin{aligned}
& t_{i j, j}+\varrho F_{i}=\varrho \ddot{u}_{i}, \\
& m_{i j, j}+\varepsilon_{i j k} t_{j k}+\varrho M_{i}=I_{i j} \ddot{\varphi}_{j} ;
\end{aligned}
$$

- the balance of the equilibrated forces:

$$
h_{i, i}+g+\varrho L=\varrho \kappa \ddot{\sigma}
$$

- the energy equation:

$$
\varrho T_{0} \dot{\eta}=q_{i, i}+\varrho S
$$

In the above equations we have used the following notations: $\varrho$-the constant mass density;

$\eta$-the specific entropy;

$T_{0}$-the constant absolute temperature of the body in its reference state;

$I_{i j}$-coefficients of microinertia;

$\kappa$-the equilibrated inertia;

$u_{i}$-the components of displacement vector;

$\varphi_{i}$-the components of microrotation vector;

$\varphi$-the volume distribution function which in the reference state is $\varphi_{0}$;

$\sigma$-the change in volume fraction measured from the reference state;

$\theta$-the temperature variation measured from the reference temperature $T_{0}$;

$\varepsilon_{i j}, \gamma_{i j}, \phi_{i}$ - the kinematic characteristics of the strain;

$t_{i j}$-the components of the stress tensor;

$m_{i j}$-the components of the couple stress tensor;

$h_{i}$-the components of the equilibrated stress vector;

$q_{i}$-the components of the heat flux vector;

$F_{i}$-the components of the body forces;

$M_{i}$-the components of the body couple;

$S$-the heat supply per unit time;

$g$-the intrinsic equilibrated force;

$L$-the extrinsic equilibrated body force;

$A_{i j m n}, B_{i j m n}, \ldots, k_{i j}$-the characteristic functions of the material, and they are prescribed functions of the spatial variable and obey the symmetry relations

$$
A_{i j m n}=A_{m n i j}, C_{i j m n}=C_{m n i j}, A_{i j}=A_{j i}, k_{i j}=k_{j i} .
$$

The entropy inequality implies

$$
k_{i j} \theta,{ }_{i} \theta,{ }_{j} \geq 0 .
$$


The equations (4) and (6) are analogous to the classical equations of motion and, respectively, to the balance equation, whereas the new balance of equilibrated force (5) can be motivated by a variational argument as in [2]. We assume that the functions coefficients $\varrho, \kappa$ and $a$ and the above constitutive coefficients are continuous differentiable functions on closure $\bar{B}$ of $B$. Moreover, we assume that $\varrho, \kappa$ and $a$ are strictly positive functions on $\bar{B}$, that is

$$
\begin{aligned}
& \varrho(x) \geq \varrho_{0}>0, \quad \varrho_{0}=\text { const } \\
& \kappa(x) \geq \kappa_{0}>0, \quad \kappa_{0}=\text { const } \\
& a(x) \geq a_{0}>0, \quad a_{0}=\text { const }
\end{aligned}
$$

The conductivity tensor $k_{i j}$ is assumed that it is symmetric, positive definite and satisfies the inequalities:

$$
k_{m} \theta_{, i} \theta_{, j} \leq k_{i j} \theta_{, i} \theta_{, j} \leq k_{M} \theta_{, i} \theta_{, j} .
$$

Here we note whit $k_{m}$ and $k_{M}$ the minimum, respectively, maximum of the conductivity tensor.

Taking into account the constitutive equation $(2)_{6}$ and the Schwarz's inequality, from (10) we obtain:

$$
\begin{aligned}
& q_{i} q_{i}=\left(k_{i j} \theta,{ }_{j}\right) q_{i} \leq\left(k_{i j} \theta_{i} \theta,_{j}\right)^{1 / 2}\left(k_{m n} q_{m} q_{n}\right)^{1 / 2} \leq \\
& \leq \quad\left(k_{i j} \theta_{i} \theta{ }_{, j}\right)^{1 / 2}\left(k_{M} q_{n} q_{n}\right)^{1 / 2}
\end{aligned}
$$

such that we can conclude that

$$
q_{i} q_{i} \leq k_{M} k_{i j} \theta_{i} \theta{ }_{j}{ }
$$

Suppose that the free energy function $\Psi$ defined in (1) is a positive definite quadratic form, that is, there exist positive constants $\mu_{m}$ and $\mu_{M}$ such that

$$
\mu_{m}\left(\varepsilon_{i j} \varepsilon_{i j}+\gamma_{i j} \gamma_{i j}+\Phi_{i} \Phi_{i}+\sigma^{2}\right) \leq 2 \Psi \leq \mu_{M}\left(\varepsilon_{i j} \varepsilon_{i j}+\gamma_{i j} \gamma_{i j}+\Phi_{i} \Phi_{i}+\sigma^{2}\right)
$$

Along with the system of equations (4) - (6) we consider the following initial conditions:

$$
\begin{aligned}
& u_{i}(x, 0)=u_{i}^{0}(x), \dot{u}_{i}(x, 0)=u_{i}^{1}(x), x \in \bar{B}, \\
& \varphi_{i}(x, 0)=\varphi_{i}^{0}(x), \dot{\varphi}_{i}(x, 0)=\varphi_{i}^{1}(x), x \in \bar{B}, \\
& \theta(x, 0)=\theta^{0}(x), \sigma(x, 0)=\sigma^{0}(x), \dot{\sigma}(x, 0)=\sigma^{1}(x), x \in \bar{B},
\end{aligned}
$$

and the following prescribed boundary conditions

$$
\begin{aligned}
& u_{i}=\bar{u}_{i} \text { on } \partial B_{1} \times[0, \infty), t_{i} \equiv t_{i j} n_{j}=\bar{t}_{i} \text { on } \partial B_{1}^{c} \times[0, \infty), \\
& \varphi_{i}=\bar{\varphi}_{i} \text { on } \partial B_{2} \times[0, \infty), m_{i} \equiv m_{i j} n_{j}=\bar{m}_{i} \text { on } \partial B_{2}^{c} \times[0, \infty), \\
& \sigma=\bar{\sigma} \text { on } \partial B_{3} \times[0, \infty), h \equiv h_{i} n_{i}=\bar{h} \text { on } \partial B_{3}^{c} \times[0, \infty), \\
& \theta=\bar{\theta} \text { on } \partial B_{4} \times[0, \infty), q \equiv q_{i} n_{i}=\bar{q} \text { on } \partial B_{4}^{c} \times[0, \infty),
\end{aligned}
$$


where $\partial B_{1}, \partial B_{2}, \partial B_{3}$ and $\partial B_{4}$ with respective complements $\partial B_{1}^{c}, \partial B_{2}^{c}, \partial B_{3}^{c}$ and $\partial B_{4}^{c}$ are subsets of $\partial B, n_{i}$ are the components of the unit outward normal to $\partial B$.

Also $u_{i}^{0}, u_{i}^{1}, \varphi_{i}^{0}, \varphi_{i}^{1}, \theta^{0}, \sigma^{0}, \sigma^{1}, \bar{u}_{i}, \bar{t}_{i}, \bar{\varphi}_{i}, \bar{m}_{i}, \bar{\sigma}, \bar{\theta}, \bar{q}$ and $\bar{h}$ are prescribed continous functions in their domains.

By a solution of the mixed initial-boundary value problem for the thermoelasticity of micropolar bodies with voids, in the cylinder $\Omega_{0}=B \times[0, \infty)$ we mean an ordered array $\left(u_{i}, \varphi_{i}, \sigma, \theta\right)$ which satisfies the equations (4)-(6) for all $(x, t) \in \Omega_{0}$, the boundary conditions (15) and the initial conditions (14). We denote by $\mathcal{P}$ the initial boundary value problem consisting of system of equations (4)-(6), the initial conditions (14) and the boundary conditions (15).

\section{Preliminary results}

We will prove some integral identities that are important in proving the results on the temporal behaviour of the solutions of the problem $\mathcal{P}$.

Theorem 1. For every solution $\left(u_{i}, \varphi_{i}, \sigma, \theta\right)$ of the problem $\mathcal{P}$ takes place the following conservation law of total energy

$$
\begin{aligned}
& \int_{B} e^{-\lambda t}\left\{\frac{1}{2}\left[\varrho \dot{u}_{i}(t) \dot{u}_{i}(t)+I_{i j} \dot{\varphi}_{i}(t) \dot{\varphi}_{j}(t)+\varrho \kappa \dot{\sigma}^{2}(t)\right]+\Psi(\mathbf{E}(t))+\frac{1}{2} a \theta^{2}(t)\right\} d V+ \\
& +\int_{0}^{t} \int_{B} e^{-\lambda s} \frac{\lambda}{2}\left[\varrho \dot{u}_{i}(s) \dot{u}_{i}(s)+I_{i j} \dot{\varphi}_{i}(s) \dot{\varphi}_{j}(s)+\varrho \kappa \dot{\sigma}^{2}(s)\right] d V d s+ \\
& +\int_{0}^{t} \int_{B} e^{-\lambda s}\left[\lambda \Psi(\mathbf{E}(s))+\frac{\lambda}{2} a \theta^{2}(s)+\frac{1}{T_{0}} k_{i j} \theta, i(s) \theta, j(s)\right] d V d s= \\
& =\int_{B}\left\{\frac{1}{2}\left[\varrho \dot{u}_{i}(0) \dot{u}_{i}(0)+I_{i j} \dot{\varphi}_{i}(0) \dot{\varphi}_{j}(0)+\varrho \kappa \dot{\sigma}^{2}(0)\right]+\Psi(\mathbf{E}(0))+\frac{1}{2} a \theta^{2}(0)\right\} d V+ \\
& +\int_{0}^{t} \int_{B} e^{-\lambda s} \varrho\left[\dot{u}_{i}(s) F_{i}(s)+\dot{\varphi}_{i}(s) M_{i}(s)+\dot{\sigma}(s) L(s)+\frac{1}{T_{0}} \theta(s) S(s)\right] d V d s+ \\
& +\int_{0}^{t} \int_{\partial B} e^{-\lambda s}\left[t_{i}(s) \dot{u}_{i}(s)+m_{i}(s) \dot{\varphi}_{i}(s)+h(s) \dot{\sigma}(s)+\frac{1}{T_{0}} q(s) \theta(s)\right] d A d s
\end{aligned}
$$

for $t \in[0, \infty)$.

Here $\lambda$ is a given positive parameter and quantities $t_{i}, m_{i}, h$ and $q$ are defined in (35).

Proof. Using the system of equations (4)-(6), the constitutive equations (2), 
the geometric relations (3) and the symmetry relations (7), one obtains

$$
\begin{aligned}
& \frac{d}{d s}\left\{\frac{1}{2}\left[\varrho \dot{u}_{i}(s) \dot{u}_{i}(s)+I_{i j} \dot{\varphi}_{i}(s) \dot{\varphi}_{j}(s)+\varrho \kappa \dot{\sigma}^{2}(s)\right]+\Psi(\mathbf{E}(s))+\frac{1}{2} a \theta^{2}(s)\right\}+ \\
& +\frac{1}{T_{0}} k_{i j} \theta, i(s) \theta, j(s)= \\
& =\varrho\left[\dot{u}_{i}(s) F_{i}(s)+\dot{\varphi}_{i}(s) M_{i}(s)+\dot{\sigma}(s) L(s)+\frac{1}{T_{0}} \theta(s) S(s)\right]+ \\
& +\left[t_{i j}(s) \dot{u}_{i}(s)+m_{i k}(s) \dot{\varphi}_{i}(s)+h_{j}(s) \dot{\sigma}(s)+\frac{1}{T_{0}} q_{j}(s) \theta(s)\right]_{, j}
\end{aligned}
$$

We multiply now in (17) by $e^{-\lambda s}$ and then integrate the obtained rezult over the cylinder $B \times[0, t]$. Because the surface $\partial B$ was assumed be smooth, we can apply the divergence theorem such that we are led to the desired result (16) and Theorem 1 is concluded.

Theorem 2. Let $\left(u_{i}, \varphi_{i}, \sigma, \theta\right)$ be a solution of the mixed initial-boundary value problem consists of the equations (4)-(6), the boundary conditions (15) and the initial conditions (14). Then we have the following identity:

$$
\begin{aligned}
& 2 \int_{B}\left[\varrho u_{i}(t) \dot{u}_{i}(t)+I_{i j} \varphi_{i}(t) \dot{\varphi}_{j}(t)+\varrho \kappa \sigma(t) \dot{\sigma}(t)+\frac{1}{T_{0}} k_{i j}\left(\int_{0}^{t} \theta, i(s) d s\right)\left(\int_{0}^{t} \theta,,_{j}(s) d s\right)\right] d V= \\
& =2 \int_{0}^{t} \int_{B}\left[\varrho \dot{u}_{i}(s) \dot{u}_{i}(s)+I_{i j} \dot{\varphi}_{i}(s) \dot{\varphi}_{j}(s)+\varrho \kappa \dot{\sigma}^{2}(s)-2 \Psi(\mathbf{E}(s))-a \theta^{2}(s)\right] d V d s+ \\
& +2 \int_{0}^{t} \int_{B} \varrho \eta(0) \theta(s) d V d s+2 \int_{B}\left[\varrho u_{i}(0) \dot{u}_{i}(0)+I_{i j} \varphi_{i}(0) \dot{\varphi}_{j}(0)+\varrho \kappa \sigma(0) \dot{\sigma}(0)\right] d V+(18) \\
& +2 \int_{0}^{t} \int_{B} \varrho\left[F_{i}(s) u_{i}(s)+M_{i}(s) \varphi_{i}(s)+L(s) \sigma(s)+\frac{1}{T_{0}} \theta(s) \int_{0}^{s} S(z) d z\right] d V d s+ \\
& 2 \int_{0}^{t} \int_{B} \varrho \eta(0) \theta(s) d V d s+2 \int_{B}\left[\varrho u_{i}(0) \dot{u}_{i}(0)+I_{i j} \varphi_{i}(0) \dot{\varphi}_{j}(0)+\varrho \kappa \sigma(0) \dot{\sigma}(0)\right] d V+ \\
& +2 \int_{0}^{t} \int_{\partial B}\left[t_{i}(s) u_{i}(s)+m_{i}(s) \varphi_{i}(s)+h(s) \sigma(s)+\frac{1}{T_{0}} \theta(s) \int_{0}^{s} q(z) d z\right] d A d s
\end{aligned}
$$

Proof. Using the motion equations $(4)_{1}$ and the geometric relations (3) one obtains,

$$
\frac{d}{d s}\left[\varrho u_{i}(s) \dot{u}_{i}(s)\right]=\varrho \dot{u}_{i}(s) \dot{u}_{i}(s)+\left[t_{j i}(s) u_{i}(s)\right]_{, j}-t_{j i}(s) u_{i, j}(s)+\varrho u_{i}(s) F_{i}(s)
$$

Also, in view of equations $(4)_{2}$ and the geometric relations (3) we are led to

$$
\begin{aligned}
& \frac{d}{d s}\left[I_{i j} \varphi_{i}(s) \dot{\varphi}_{i}(s)\right]=I_{i j} \dot{\varphi}_{i}(s) \dot{\varphi}_{i}(s)+\left[m_{j i}(s) \varphi_{i}(s)\right]_{, j}- \\
& -m_{j i}(s) \varphi_{i, j}(s)+\varepsilon_{i j k} t_{j k}(s) \varphi_{i}(s)+\varrho \varphi_{i}(s) M_{i}(s)
\end{aligned}
$$


By adding the relations (19) and (20) we arrive at equality

$$
\begin{aligned}
& \frac{d}{d s}\left[\varrho u_{i}(s) \dot{u}_{i}(s)+I_{i j} \varphi_{i}(s) \dot{\varphi}_{j}(s)\right]=\varrho \dot{u}_{i}(s) \dot{u}_{i}(s)+I_{i j} \dot{\varphi}_{i}(s) \dot{\varphi}_{i}(s)+ \\
& +\left[t_{j i}(s) u_{i}(s)+m_{j i}(s) \varphi_{i}(s)\right]_{, j}-t_{i j}(s) \varepsilon_{i j}(s)-m_{i j}(s) \gamma_{i j}(s)
\end{aligned}
$$

With the aid of the constitutive equation $(2)_{1}$ we can write:

$$
\begin{aligned}
& t_{i j}(s) \varepsilon_{i j}(s)=A_{i j m n} \varepsilon_{i j}(s) \varepsilon_{m n}(s)+B_{i j m n} \varepsilon_{i j}(s) \gamma_{m n}(s)+2 B_{i j} \sigma(s) \varepsilon_{i j}(s)+ \\
& +2 D_{i j k} \phi_{k}(s) \varepsilon_{i j}(s)-\left[B_{i j} \sigma(s) \varepsilon_{i j}(s)+D_{i j k} \phi_{k}(s) \varepsilon_{i j}(s)+\alpha_{i j} \theta(s) \varepsilon_{i j}(s)\right](22)
\end{aligned}
$$

Analogous, with the aid of the constitutive equation $(2)_{2}$ we can write:

$$
\begin{aligned}
& m_{i j}(s) \gamma_{i j}(s)=B_{m n i j} \varepsilon_{i j}(s) \gamma_{m n}(s)+C_{i j m n} \gamma_{i j}(s) \gamma_{m n}(s)+2 C_{i j} \sigma(s) \gamma_{i j}(s)+ \\
& +2 E_{i j k} \phi_{k}(s) \gamma_{i j}(s)-\left[C_{i j} \sigma(s) \gamma_{i j}(s)+E_{i j k} \phi_{k}(s) \gamma_{i j}(s)+\beta_{i j} \theta(s) \gamma_{i j}(s)\right]
\end{aligned}
$$

By adding relations (22) and (23) together, we obtain

$$
\begin{aligned}
& t_{i j}(s) \varepsilon_{i j}(s)+m_{i j}(s) \gamma_{i j}(s)=A_{i j m n} \varepsilon_{i j}(s) \varepsilon_{m n}(s)+ \\
& +2 B_{m n i j} \varepsilon_{i j}(s) \gamma_{m n}(s)+C_{i j m n} \gamma_{i j}(s) \gamma_{m n}(s)+2 B_{i j} \sigma(s) \varepsilon_{i j}(s)+ \\
& +2 D_{i j k} \phi_{k}(s) \varepsilon_{i j}(s)+2 C_{i j} \sigma(s) \gamma_{i j}(s)+2 E_{i j k} \phi_{k}(s) \gamma_{i j}(s)- \\
& -\left[B_{i j} \sigma(s) \varepsilon_{i j}(s)+D_{i j k} \phi_{k}(s) \varepsilon_{i j}(s)+\alpha_{i j} \theta(s) \varepsilon_{i j}(s)\right]- \\
& -\left[C_{i j} \sigma(s) \gamma_{i j}(s)+E_{i j k} \phi_{k}(s) \gamma_{i j}(s)+\beta_{i j} \theta(s) \gamma_{i j}(s)\right]
\end{aligned}
$$

For the last two parentheses in (24) we find equivalent expressions if we use formulas $(2)_{3}-(2)_{5}$ and $(3)$

$$
\begin{gathered}
\quad\left[B_{i j} \varepsilon_{i j}(s)+C_{i j} \gamma_{i j}(s)\right] \sigma(s)+\left[D_{i j k} \varepsilon_{i j}(s)+E_{i j k} \gamma_{i j}(s)\right] \phi_{k}(s)+ \\
+\left[\alpha_{i j} \varepsilon_{i j}(s)+\beta_{i j} \gamma_{i j}(s)\right] \theta(s)=g(s) \sigma(s)-\xi \sigma^{2}(s)-2 d_{i} \phi_{i}(s) \sigma(s)+(25) \\
{\left[h_{i}(s) \sigma(s)\right]_{, i}-h_{i, i}(s) \sigma(s)-A_{i j} \phi_{i}(s) \phi_{j}(s)-a \theta^{2}(s)+\varrho \eta(s) \theta(s)}
\end{gathered}
$$

Now let's integrate the energy equation (6)

$$
\varrho \eta(s)=\frac{1}{T_{0}} \int_{0}^{s} q_{i, i}(z) d z+\frac{\varrho}{T_{0}} \int_{0}^{s} S(z) d z+\varrho \eta(0)
$$

In view of equation (5) and relation (26) we are led to

$$
\begin{aligned}
& {\left[g(s)+h_{i, i}(s)\right] \sigma(s)-\varrho \eta(s) \theta(s)=[\varrho \kappa \ddot{\sigma}(s)-\varrho L(s)] \sigma(s)-\varrho \eta(0) \theta(s)-} \\
& -\frac{\varrho}{T_{0}} \int_{0}^{s} S(z) d z-\left[\frac{1}{T_{0}} \theta(s) \int_{0}^{s} q_{i}(z) d z\right]_{, i}+\frac{1}{T_{0}} \theta, i(s) \int_{0}^{s} q_{i, i}(z) d z \quad(27)
\end{aligned}
$$


With the aid of constitutive equation $(2)_{6}$, the equality (27) can be restated in the form

$$
\begin{aligned}
& {\left[g(s)+h_{i, i}(s)\right] \sigma(s)-\varrho \eta(s) \theta(s)=-\varrho \kappa \dot{\sigma}^{2}(s)-\varrho \eta(0) \theta(s)+} \\
& +\frac{d}{d s}\left[\varrho \kappa \sigma(s) \dot{\sigma}(s)+\frac{1}{2 T_{0}} k_{i j}\left(\int_{0}^{s} \theta, i(z) d z\right)\left(\int_{0}^{s} \theta,{ }_{j}(z) d z\right)\right]- \\
& -\varrho\left[L(s) \sigma(s)+\frac{1}{T_{0}} \theta(s) \int_{0}^{s} S(z) d z\right]-\left[\frac{1}{T_{0}} \theta(s) \int_{0}^{s} q_{i}(z) d z\right]_{, i}
\end{aligned}
$$

Now, we replace the relations (24), (25) and (28) into equality (21) so that we can obtain

$$
\begin{aligned}
& \frac{d}{d s}\left[2 \varrho u_{i}(s) \dot{u}_{i}(s)+2 I_{i j} \varphi_{i}(s) \dot{\varphi}_{j}(s)+2 \varrho \kappa \sigma(s) \dot{\sigma}(s)+\frac{1}{T_{0}} k_{i j}\right. \\
& \left.\left(\int_{0}^{s} \theta,{ }_{i}(z) d z\right)\left(\int_{0}^{s} \theta_{, j}(z) d z\right)\right]= \\
& =2 \varrho \dot{u}_{i}(s) \dot{u}_{i}(s)+2 I_{i j} \dot{\varphi}_{i}(s) \dot{\varphi}_{j}(s)+2 \varrho \kappa \dot{\sigma}^{2}(s)-2\left[2 \Psi(\mathbf{E}(s))+a \theta^{2}(s)\right]+ \\
& +2 \varrho\left[F_{i}(s) u_{i}(s)+M_{i}(s) \varphi_{i}(s)+L(s) \sigma(s)+\frac{1}{T_{0}} \theta(s) \int_{0}^{s} S(z) d z\right]+(29) \\
& +2\left[t_{j i}(s) u_{i}(s)+m_{j i}(s) \varphi_{i}(s)+h_{j}(s) \sigma(s)+\frac{1}{T_{0}} \theta(s) \int_{0}^{s} q_{j}(z) d z\right]_{, j}+ \\
& +2 \varrho \eta(0) \theta(s) .
\end{aligned}
$$

Finally, we integrate the equality (29) onto the cylinder $B \times[0, t]$ then apply the divergence theorem so that we get to the desired identity (18) such as the proof of Theorem 2 is finished.

Theorem 3. Let $\left(u_{i}, \varphi_{i}, \sigma, \theta\right)$ be a solution of the mixed initial-boundary value problem $\mathcal{P}$. Then take place the following identity:

$$
\begin{aligned}
& 2 \int_{B}\left[\varrho u_{i}(t) \dot{u}_{i}(t)+I_{i j} \varphi_{i}(t) \dot{\varphi}_{j}(t)+\varrho \kappa \sigma(t) \dot{\sigma}(t)+\frac{1}{T_{0}} k_{i j}\right. \\
& \left.\quad\left(\int_{0}^{t} \theta,{ }_{i}(s) d s\right)\left(\int_{0}^{t} \theta,{ }_{j}(s) d s\right)\right] d V= \\
& =\int_{B}\left\{\varrho\left[u_{i}(0) \dot{u}_{i}(2 t)+\dot{u}_{i}(0) u_{i}(2 t)\right]+I_{i j}\left[\varphi_{i}(0) \dot{\varphi}_{j}(2 t)+\dot{\varphi}_{j}(0) \varphi_{i}(2 t)\right]\right\} d V+ \\
& +\int_{B} \varrho \kappa[\sigma(0) \dot{\sigma}(2 t)+\dot{\sigma}(0) \sigma(2 t)] d V+\int_{0}^{t} \int_{B} \varrho \eta(0)[\theta(t-s)-\theta(t+s)] d V d s+ \\
& +\int_{0}^{t} \int_{B} \varrho\left[u_{i}(t+s) F_{i}(t-s)-u_{i}(t-s) F_{i}(t+s)\right] d V d s+
\end{aligned}
$$




$$
\begin{aligned}
& +\int_{0}^{t} \int_{B} I_{i j}\left[\varphi_{i}(t+s) M_{i}(t-s)-\varphi_{i}(t-s) M_{i}(t+s)\right] d V d s+ \\
& +\int_{0}^{t} \int_{B}[\sigma(t+s) L(t-s)-\sigma(t-s) L(t+s)] d V d s+ \\
& +\int_{0}^{t} \int_{B} \frac{1}{T_{0}}\left[\theta(t-s) \int_{0}^{t+s} S(z) d z-\theta(t+s) \int_{0}^{t-s} S(z) d z\right] d V d s+ \\
& +\int_{0}^{t} \int_{\partial B}\left[u_{i}(t+s) t_{i}(t-s)-u_{i}(t-s) t_{i}(t+s)\right] d A d s+ \\
& +\int_{0}^{t} \int_{\partial B}\left[\varphi_{i}(t+s) m_{i}(t-s)-\varphi_{i}(t-s) m_{i}(t+s)\right] d A d s+ \\
& +\int_{0}^{t} \int_{\partial B}[\sigma(t+s) h(t-s)-\sigma(t-s) h(t+s)] d A d s+ \\
& +\int_{0}^{t} \int_{\partial B} \frac{1}{T_{0}}\left[\theta(t-s) \int_{0}^{t+s} q(z) d z-\theta(t+s) \int_{0}^{t-s} q(z) d z\right] d A d s
\end{aligned}
$$

Proof. It is no difficult to observe that

$$
\begin{aligned}
& -\frac{d}{d s}\left\{\varrho\left[u_{i}(t+s) \dot{u}_{i}(t-s)+\dot{u}_{i}(t+s) u_{i}(t-s)\right]\right\}= \\
& =\varrho\left[u_{i}(t+s) \ddot{u}_{i}(t-s)-u_{i}(t-s) \ddot{u}_{i}(t+s)\right], s \in[0, t], t \in[0, \infty)(31)
\end{aligned}
$$

Taking into account the equations of motion $(4)_{1}$, the right side term from (31) can be rewrite in the form

$$
\begin{aligned}
& \varrho\left[u_{i}(t+s) \ddot{u}_{i}(t-s)-u_{i}(t-s) \ddot{u}_{i}(t+s)\right]= \\
& =\varrho\left[u_{i}(t+s) F_{i}(t-s)-u_{i}(t-s) F_{i}(t+s)\right]+ \\
& +\left[u_{i}(t+s) t_{j i}(t-s)-u_{i}(t-s) t_{j i}(t+s)\right], j+ \\
& +\left[u_{i, j}(t-s) t_{j i}(t+s)-u_{i, j}(t+s) t_{j i}(t-s)\right]
\end{aligned}
$$

Hence, taking into account the relation (32), the identity (31) received the form

$$
\begin{aligned}
& -\frac{d}{d s}\left\{\varrho\left[u_{i}(t+s) \dot{u}_{i}(t-s)+\dot{u}_{i}(t+s) u_{i}(t-s)\right]\right\}= \\
& =\varrho\left[u_{i}(t+s) F_{i}(t-s)-u_{i}(t-s) F_{i}(t+s)\right]+ \\
& +\left[u_{i}(t+s) t_{j i}(t-s)-u_{i}(t-s) t_{j i}(t+s)\right]_{, j}+ \\
& +\left[u_{i, j}(t-s) t_{j i}(t+s)-u_{i, j}(t+s) t_{j i}(t-s)\right]
\end{aligned}
$$


Clarly, we have

$$
\begin{gathered}
-\frac{d}{d s}\left\{I_{i j}\left[\varphi_{i}(t+s) \dot{\varphi}_{j}(t-s)+\dot{\varphi}_{i}(t+s) \varphi_{i}(t-s)\right]\right\}= \\
=I_{i j}\left[\varphi_{i}(t+s) \ddot{\varphi}_{i}(t-s)-\varphi_{i}(t-s) \ddot{\varphi}_{i}(t+s)\right], s \in[0, t], t \in[0, \infty)(34)
\end{gathered}
$$

Taking into account the equations of motion $(4)_{2}$, the right side term from (34) can be rewrite in the form

$$
\begin{aligned}
& I_{i j}\left[\varphi_{i}(t+s) \ddot{\varphi}_{i}(t-s)-\varphi_{i}(t-s) \ddot{\varphi}_{i}(t+s)\right]= \\
& =\varrho\left[\varphi_{i}(t+s) M_{i}(t-s)-\varphi_{i}(t-s) M_{i}(t+s)\right]+ \\
& +\left[\varphi_{i}(t+s) m_{j i}(t-s)-\varphi_{i}(t-s) m_{j i}(t+s)\right],{ }_{j}+ \\
& +\left[\varphi_{i, j}(t-s) m_{j i}(t+s)-\varphi_{i, j}(t+s) m_{j i}(t-s)\right]+ \\
& +\varepsilon_{i j k}\left[\varphi_{i}(t+s) t_{j k}(t-s)-\varphi_{i}(t-s) t_{j k}(t+s)\right]
\end{aligned}
$$

Hence, taking into account the relation (35), the identity (34) received the form

$$
\begin{aligned}
& -\frac{d}{d s}\left\{I_{i j}\left[\varphi_{i}(t+s) \dot{\varphi}_{j}(t-s)+\dot{\varphi}_{i}(t+s) \varphi_{i}(t-s)\right]\right\}= \\
& =\varrho\left[\varphi_{i}(t+s) M_{i}(t-s)-\varphi_{i}(t-s) M_{i}(t+s)\right]+ \\
& +\left[\varphi_{i}(t+s) m_{j i}(t-s)-\varphi_{i}(t-s) m_{j i}(t+s)\right]_{, j}+ \\
& +\left[\varphi_{i, j}(t-s) m_{j i}(t+s)-\varphi_{i, j}(t+s) m_{j i}(t-s)\right]+ \\
& +\varepsilon_{i j k}\left[\varphi_{i}(t+s) t_{j k}(t-s)-\varphi_{i}(t-s) t_{j k}(t+s)\right]
\end{aligned}
$$

Now, we add relations (36) and (33) term by term and by using the geometric relations (3) we are led to

$$
\begin{aligned}
& -\frac{d}{d s}\left\{\varrho\left[u_{i}(t+s) \dot{u}_{i}(t-s)+\dot{u}_{i}(t+s) u_{i}(t-s)\right]\right\}+ \\
& -\frac{d}{d s}\left\{I_{i j}\left[\varphi_{i}(t+s) \dot{\varphi}_{j}(t-s)+\dot{\varphi}_{i}(t+s) \varphi_{i}(t-s)\right]\right\}= \\
& =\varrho\left[u_{i}(t+s) F_{i}(t-s)-u_{i}(t-s) F_{i}(t+s)\right]+ \\
& +\varrho\left[\varphi_{i}(t+s) M_{i}(t-s)-\varphi_{i}(t-s) M_{i}(t+s)\right]+ \\
& +\left[u_{i}(t+s) t_{j i}(t-s)-u_{i}(t-s) t_{j i}(t+s)\right]_{, j}+ \\
& +\left[\varphi_{i}(t+s) m_{j i}(t-s)-\varphi_{i}(t-s) m_{j i}(t+s)\right]_{, j}+ \\
& +\left[t_{i j}(t+s) \varepsilon_{i j}(t-s)-t_{i j}(t-s) \varepsilon_{i j}(t+s)\right]+ \\
& +\left[m_{i j}(t+s) \gamma_{i j}(t-s)-m_{i j}(t-s) \gamma_{i j}(t+s)\right]
\end{aligned}
$$


Let us find another form for the last two parenthesis from equality (37). By using the constitutive equations $(2)_{1}-(2)_{5}$ we deduce

$$
\begin{aligned}
& {\left[t_{i j}(t+s) \varepsilon_{i j}(t-s)-t_{i j}(t-s) \varepsilon_{i j}(t+s)\right]+} \\
& +\left[m_{i j}(t+s) \gamma_{i j}(t-s)-m_{i j}(t-s) \gamma_{i j}(t+s)\right]= \\
& =[\sigma(t-s) g(t+s)-\sigma(t+s) g(t-s)]+ \\
& +\left[h_{i}(t-s) \phi(t+s)-h_{i}(t+s) \phi(t-s)\right]+ \\
& +\varrho[\theta(t-s) \eta(t+s)-\theta(t+s) \eta(t-s)]
\end{aligned}
$$

Taking into account the balance of the equilibrated forces (5) and the geometric equations (3) we obtain

$$
\begin{aligned}
& h_{i}(t-s) \phi(t+s)-h_{i}(t+s) \phi(t-s)= \\
& =\left[h_{i}(t-s) \sigma(t+s)-h_{i}(t+s) \sigma(t-s)\right], i \\
& +[\sigma(t+s) g(t-s)-\sigma(t-s) g(t+s)]+ \\
& +\varrho[\sigma(t+s) L(t-s)-\sigma(t-s) L(t+s)]+ \\
& +\varrho \kappa[\sigma(t-s) \ddot{\sigma}(t+s)-\sigma(t+s) \ddot{\sigma}(t-s)]
\end{aligned}
$$

Also, by using the equation of energy (6) we deduce

$$
\begin{aligned}
& \varrho[\theta(t-s) \eta(t+s)-\theta(t+s) \eta(t-s)]=\varrho \eta(0)[\theta(t-s)-\theta(t+s)]+ \\
& +\frac{\varrho}{T_{0}}\left[\theta(t-s) \int_{0}^{t+s} S(z) d z-\theta(t+s) \int_{0}^{t-s} S(z) d z\right]+ \\
& +\frac{1}{T_{0}}\left[\theta(t-s) \int_{0}^{t+s} q_{i}(z) d z-\theta(t+s) \int_{0}^{t-s} q_{i}(z) d z\right]_{, i}+ \\
& +\frac{1}{T_{0}} k_{i j}\left[\theta,{ }_{i}(t+s) \int_{0}^{t-s} \theta,{ }_{j}(z) d z-\theta,{ }_{i}(t-s) \int_{0}^{t+s} \theta_{i}(z) d z\right]
\end{aligned}
$$

We substitute equalities (40) and (39) into (38) and then the resulting equality is introduced in (37). Hence, we obtain

$$
\begin{aligned}
& -\frac{d}{d s}\left\{\varrho\left[u_{i}(t+s) \dot{u}_{i}(t-s)+\dot{u}_{i}(t+s) u_{i}(t-s)\right]\right\}- \\
& -\frac{d}{d s}\left\{I_{i j}\left[\varphi_{i}(t+s) \dot{\varphi}_{j}(t-s)+\dot{\varphi}_{i}(t+s) \varphi_{i}(t-s)\right]\right\}- \\
& -\frac{d}{d s}\{\varrho \kappa[\sigma(t-s) \dot{\sigma}(t+s)+\sigma(t+s) \dot{\sigma}(t-s)]\}- \\
& -\frac{d}{d s}\left[\frac{1}{T_{0}} k_{i j}\left(\int_{0}^{t+s} \theta, i(z) d z\right)\left(\int_{0}^{t-s} \theta, j(z) d z\right)\right]=
\end{aligned}
$$




$$
\begin{aligned}
& =\varrho\left[u_{i}(t+s) F_{i}(t-s)-u_{i}(t-s) F_{i}(t+s)\right]+ \\
& +\varrho\left[\varphi_{i}(t+s) M_{i}(t-s)-\varphi_{i}(t-s) M_{i}(t+s)\right]+ \\
& +\varrho[\sigma(t+s) L(t-s)-\sigma(t-s) L(t+s)]+ \\
& +\frac{\varrho}{T_{0}}\left[\theta(t-s) \int_{0}^{t+s} S(z) d z-\theta(t+s) \int_{0}^{t-s} S(z) d z\right]+ \\
& +\varrho \eta(0)[\theta(t-s)-\theta(t+s)]+ \\
& +\left[u_{i}(t+s) t_{j i}(t-s)-u_{i}(t-s) t_{j i}(t+s)\right]_{, j}+ \\
& +\left[\varphi_{i}(t+s) m_{j i}(t-s)-\varphi_{i}(t-s) m_{j i}(t+s)\right]_{, j}+ \\
& +\left[h_{j}(t-s) \sigma(t+s)-h_{j}(t+s) \sigma(t-s)\right]_{, j}+ \\
& +\frac{1}{T_{0}}\left[\theta(t-s) \int_{0}^{t+s} q_{j}(z) d z-\theta(t+s) \int_{0}^{t-s} q_{j}(z) d z\right]_{, j}
\end{aligned}
$$

Finally, we integrate the equality (41) over cylinder $B \times[0, t]$ and, after we use the divergence theorem, the desired identity (30) is obtained such that the proof of Theorem 3 is complete.

\section{Temporal behaviour of solutions}

In order to prove the main results of this study, that is, the temporal behaviour of solutions of the problem $\mathcal{P}$, defined at the end of Section 2, we need other preliminary results.

Assume that the boundary of $B$, denoted by $\partial B$, is a sufficiently smooth surface to admit the aplication of divergence theorem. Also, we denote the closure of $B$ by $\bar{B}$.

We study the temporal behaviour of solutions of problem $\mathcal{P}$, in the case of null boundary data and null body supplies.

Consider the problem $\mathcal{P}_{0}$ defined by the constitutive equations (2), the geometric equations (3), the equations of motion

$$
\begin{aligned}
& t_{i j, j}=\varrho \ddot{u}_{i}, \\
& m_{i j, j}+\varepsilon_{i j k} t_{j k}=I_{i j} \ddot{\varphi}_{j} ; \\
& h_{i, i}+g=\varrho \kappa \ddot{\sigma} ; \\
& \varrho T_{0} \dot{\eta}=q_{i, i}
\end{aligned}
$$


the boundary conditions

$$
\begin{aligned}
& u_{i}=0 \text { on } \partial B_{1} \times[0, \infty), t_{i} \equiv t_{i j} n_{j}=0 \text { on } \partial B_{1}^{c} \times[0, \infty), \\
& \varphi_{i}=0 \text { on } \partial B_{2} \times[0, \infty), m_{i} \equiv m_{i j} n_{j}=0 \text { on } \partial B_{2}^{c} \times[0, \infty), \\
& \sigma=0 \text { on } \partial B_{3} \times[0, \infty), h \equiv h_{i} n_{i}=0 \text { on } \partial B_{3}^{c} \times[0, \infty), \\
& \theta=0 \text { on } \partial B_{4} \times[0, \infty), q \equiv q_{i} n_{i}=0 \text { on } \partial B_{4}^{c} \times[0, \infty),
\end{aligned}
$$

and the initial conditions in the form (14).

Consider $\left(u_{i}, \varphi_{i}, \sigma, \theta\right)$ a solution of problem $\mathcal{P}_{0}$ and introduce the Cesaro means for all energy components:

1. Cesaro mean of kinetic energy:

$$
\mathcal{K}=\frac{1}{2 t} \int_{0}^{t} \int_{B}\left[\varrho \dot{u}_{i}(s) \dot{u}_{i}(s)+I_{i j} \dot{\varphi}_{i}(s) \dot{\varphi}_{i}(s)+\varrho \kappa \dot{\sigma}^{2}(s)\right] d V d s
$$

2. Cesaro mean of strain energy:

$$
\begin{aligned}
& \mathcal{S}=\frac{1}{2 t} \int_{0}^{t} \int_{B}\left[A_{i j m n} \varepsilon_{i j}(s) \varepsilon_{m n}(s)+2 B_{i j m n} \varepsilon_{i j}(s) \gamma_{m n}(s)+\right. \\
& +C_{i j m n} \gamma_{i j}(s) \gamma_{m n}(s)+2 B_{i j} \sigma(s) \varepsilon_{i j}(s)+2 C_{i j} \sigma(s) \gamma_{i j}(s)+ \\
& +2 D_{i j k} \phi_{k}(s) \varepsilon_{i j}(s)++2 E_{i j k} \phi_{k}(s) \gamma_{i j}(s)+2 d_{i} \sigma(s) \phi_{i}(s)+ \\
& \left.+2 \xi \sigma^{2}(s)+A_{i j} \phi_{i}(s) \phi_{j}(s)-\omega \dot{\sigma}^{2}(s)\right] d V d s
\end{aligned}
$$

3. Cesaro mean of thermal energy:

$$
\mathcal{T}=\frac{1}{2 t} \int_{0}^{t} \int_{B} a \theta^{2}(s) d V d s
$$

4. Cesaro mean of energy of diffusion:

$$
\mathcal{T}=\frac{1}{t} \int_{0}^{t} \int_{0}^{s} \int_{B} \frac{1}{T_{0}} k_{i j} \theta, i(z) \theta_{j}(z) d V d z d s
$$

In the case meas $\left(\partial B_{1}\right)=0$ there exists a family of rigid displacements, rigid microrotations and null temperature and null change in volume fraction that satisfy the equations (2), (3)and (42) and the boundary conditions (43). Thus, we can decompose the initial data as follows

$$
\begin{aligned}
& u_{i}^{0}=u_{i}^{*}+U_{i}^{0}, u_{i}^{1}=\dot{u}_{i}^{*}+\dot{U}_{i}^{0} \\
& \varphi_{i}^{0}=\varphi_{i}^{*}+\Phi_{i}^{0}, \varphi_{i}^{1}=\dot{\varphi}_{i}^{*}+\dot{\Phi}_{i}^{0}
\end{aligned}
$$

where the rigid displacements $u_{i}^{*}$ and $\dot{u}_{i}^{*}$ and the rigid microrotations $\varphi_{i}^{*}$ and $\dot{\varphi}_{i}^{*}$ are determined such that

$$
\begin{aligned}
& \int_{B} \varrho U_{i}^{0} d V=0, \int_{B} \varrho \varepsilon_{i j k} x_{j} U_{k}^{0} d V=0, \int_{B} \varrho \dot{U}_{i}^{0} d V=0, \\
& \int_{B} \varrho \varepsilon_{i j k} x_{j} \dot{U}_{k}^{0} d V=0, \int_{B} I_{i j} \Phi_{j}^{0} d V=0, \int_{B} I_{i j} \dot{\Phi}_{j}^{0} d V=0,
\end{aligned}
$$


where, as usual, $\varepsilon_{i j k}$ is Ricci's symbol.

We believe that the following are common notations

$$
\begin{aligned}
& \hat{\mathbf{C}}^{1}(B)=\left\{\mathbf{v}=\left(u_{1}, u_{2}, u_{3}, \varphi_{1}, \varphi_{2}, \varphi_{3}\right), u_{i} \in C^{1}(\bar{B}),\right. \\
& \left.\varphi_{i} \in C^{1}(\bar{B}): u_{i}=0 \text { on } \partial B_{1}, \varphi_{i}=0 \text { on } \partial B_{2}\right\} \\
& \hat{C}^{1}(B)=\left\{\sigma \in C^{1}(\bar{B}): \sigma=0 \text { on } \partial B_{3}\right\} \\
& \tilde{C}^{1}(B)=\left\{\theta \in C^{1}(\bar{B}): \theta=0 \text { on } \partial B_{4}\right\} \\
& \hat{\mathbf{W}}^{1}(B)=\text { the complection of } \hat{\mathbf{C}}^{1}(B) \text { by means of }\|\cdot\|_{\mathbf{W}^{1}(B)} \\
& \hat{W}^{1}(B)=\text { the complection of } \hat{C}^{1}(B) \text { by means of }\|\cdot\|_{W^{1}(B)} \\
& \tilde{W}^{1}(B)=\text { the complection of } \tilde{C}^{1}(B) \text { by means of }\|\cdot\|_{W^{1}(B)}
\end{aligned}
$$

As is well known, $C^{1}(\bar{B})$ is the notation for the set of scalar continuously differentiable functions on $\bar{B}$ and $W^{1}(B)$ represents the familiar Sobolev space [10]. Also, we used the notation $\mathbf{W}^{1}(B)=\left[W^{1}(B)\right]^{6}$.

Based on hypothesis (13) we obtain the following inequality, of Korn type, [11]

$$
\begin{aligned}
& \frac{1}{2} \int_{B}\left[A_{i j m n} \varepsilon_{i j} \varepsilon_{m n}+2 B_{i j m n} \varepsilon_{i j} \gamma_{m n}+\right. \\
& \left.C_{i j m n} \gamma_{i j} \gamma_{m n}\right] d V \geq m_{1} \int_{B}\left[u_{i} u_{i}+\varphi_{i} \varphi_{i}\right] d V,
\end{aligned}
$$

for any $\mathbf{v} \in \hat{\mathbf{W}}^{1}(B)$. Here $m_{1}$ is a positive constant.

Also, taking into account the hypothesis (10) we deduce that there exists a positive constant $m_{2}$ such that the following Poincare's type inequality holds

$$
\frac{1}{2} \int_{B} k_{i j} \theta,{ }_{i} \theta,{ }_{j} d V \geq m_{2} \int_{B} \theta^{2} d V,
$$

for any $\theta \in \hat{W}^{1}(B)$.

In the case meas $\left(\partial B_{1}\right)=0$ and meas $\left(\partial B_{2}\right)=0$ we decompose the solution $\left(u_{i}, \varphi_{i}, \sigma, \theta\right)$ as follows

$$
u_{i}=u_{i}^{*}+t \dot{u}_{i}^{*}+v_{i}, \varphi_{i}=\varphi_{i}^{*}+t \dot{\varphi}_{i}^{*}+\chi_{i}, \sigma=\zeta, \theta=\gamma
$$

where $\left(v_{i}, \chi_{i}, \zeta, \gamma\right) \in \hat{\mathbf{W}}^{1}(B) \times \hat{W}^{1}(B) \times \tilde{W}^{1}(B)$ is the solution of the problem $\mathcal{P}_{0}$ which corresponds to the following initial conditions

$$
v_{i}=U_{i}^{0}, \dot{v}_{i}=\dot{U}_{i}^{0}, \chi_{i}=\Phi_{i}^{0}, \dot{\chi}_{i}=\dot{\Phi}_{i}^{0}, \zeta=\sigma^{0}, \gamma=\theta^{0}, \text { at } t=0
$$

We will use in what follows the total energy defined by

$$
\begin{aligned}
& \mathcal{E}=\frac{1}{2} \int_{B}\left[\varrho \dot{u}_{i}(t) \dot{u}_{i}(t)+I_{i j} \dot{\varphi}_{i}(t) \varphi_{j}(t)+\varrho \kappa \dot{\sigma}^{2}(t)+2 \Psi(\mathbf{E}(t))+a \theta^{2}(t)\right] d V+ \\
& +\int_{0}^{t} \int_{B} \frac{1}{T_{0}} k_{i j} \theta,{ }_{i}(z) \theta,{ }_{j}(z) d V d z .
\end{aligned}
$$


Now we have everything ready for the proof of the asymptotic partition of total energy, with the help of Cesaro means. This will be done in the following theorem.

Theorem 4. Consider a solution $\left(u_{i}, \varphi_{i}, \sigma, \theta\right)$ of the initial boundary value problem $\mathcal{P}_{0}$. If we suppose that

$$
\begin{aligned}
& \left(u_{i}^{0}, \varphi_{i}^{0}\right) \in \mathbf{W}_{1}(B),\left(u_{i}^{1}, \varphi_{i}^{1}\right) \in \mathbf{W}_{0}(B), \\
& \left(\sigma^{0}, \theta^{0}\right) \in W_{1}(B) \times W_{1}(B), \sigma^{1} \in W_{0}(B),
\end{aligned}
$$

then take place the following relation

$$
\lim _{t \rightarrow \infty} \mathcal{T}(t)=0 .
$$

Also, we have

i. If meas $\left(\partial B_{1}\right) \neq 0$ and meas $\left(\partial B_{2}\right) \neq 0$, then

$$
\begin{aligned}
& \lim _{t \rightarrow \infty} \mathcal{T}(t)=\lim _{t \rightarrow \infty} \mathcal{S}(t) \\
& \lim _{t \rightarrow \infty} \mathcal{D}(t)=\mathcal{E}(0)-2 \lim _{t \rightarrow \infty} \mathcal{K}(t)=\mathcal{E}(0)-2 \lim _{t \rightarrow \infty} \mathcal{S}(t)
\end{aligned}
$$

ii. If meas $\left(\partial B_{1}\right)=0$ and meas $\left(\partial B_{2}\right)=0$, then

$$
\begin{aligned}
& \lim _{t \rightarrow \infty} \mathcal{K}(t)=\lim _{t \rightarrow \infty} \mathcal{S}(t)+\frac{1}{2} \int_{B}\left[\varrho \dot{u}_{i}^{*} \dot{u}_{i}^{*}+I_{i j} \dot{\varphi}_{i}^{*} \varphi_{j}^{*}\right] d V \\
& \lim _{t \rightarrow \infty} \mathcal{D}(t)=\mathcal{E}(0)-2 \lim _{t \rightarrow \infty} \mathcal{K}(t)+\frac{1}{2} \int_{B}\left[\varrho \dot{u}_{i}^{*} \dot{u}_{i}^{*}+I_{i j} \dot{\varphi}_{i}^{*} \varphi_{j}^{*}\right] d V= \\
& =\mathcal{E}(0)-2 \lim _{t \rightarrow \infty} \mathcal{S}(t)+\frac{1}{2} \int_{B}\left[\varrho \dot{u}_{i}^{*} \dot{u}_{i}^{*}+I_{i j} \dot{\varphi}_{i}^{*} \varphi_{j}^{*}\right] d V
\end{aligned}
$$

Proof. We use equality (16) in which we replace $\lambda$ with zero. Then keep in mind that $\left(u_{i}, \varphi_{i}, \sigma, \theta\right)$ is a solution of problem $\mathcal{P}_{0}$ and the definition from (54) of total energy $\mathcal{E}$. Thus obtain that

$$
\mathcal{E}(t)=\mathcal{E}(0), t \geq 0 .
$$

Now replace the total energy components defined in relations (44)-(47) into conservation law (60) such that we obtain

$$
\mathcal{K}(t)+\mathcal{S}(t)+\mathcal{T}(t)+\mathcal{D}(t)=\mathcal{E}(0), \text { for all } t>0 .
$$


If we use equalities (18) and (30) and take into account the fact that $\left(u_{i}, \varphi_{i}, \sigma, \theta\right)$ is a solution of problem $\mathcal{P}_{0}$, then we are led to the relation

$$
\begin{aligned}
& \int_{0}^{t} \int_{B}\left[\varrho \dot{u}_{i}(t) \dot{u}_{i}(t)+I_{i j} \dot{\varphi}_{i}(t) \varphi_{j}(t)+\varrho \kappa \dot{\sigma}^{2}(t)-2 \Psi(\mathbf{E}(t))-a \theta^{2}(t)\right] d V d s= \\
& =-\int_{B}\left[\varrho u_{i}(0) \dot{u}_{i}(0)+I_{i j} \varphi_{i}(0) \dot{\varphi}_{j}(0)+\varrho \kappa \sigma(0) \dot{\sigma}(0)\right] d V+ \\
& +\int_{B}\left\{\varrho\left[u_{i}(0) \dot{u}_{i}(2 t)+\dot{u}_{i}(0) u_{i}(2 t)\right]+I_{i j}\left[\varphi_{i}(0) \dot{\varphi}_{j}(2 t)+\varphi_{i}(2 t) \dot{\varphi}_{j}(0)\right]+(62)\right. \\
& +\varrho \kappa[\sigma(0) \dot{\sigma}(2 t)+\sigma(2 t) \dot{\sigma}(0)]\} d V-2 \int_{0}^{t} \int_{B} \varrho \eta(0) \theta(s) d V d s+ \\
& +\int_{0}^{t} \int_{B} \varrho \eta(0)[\theta(t-s)-\theta(t+s)] d V d s
\end{aligned}
$$

for $t \geq 0$.

Using relations (44)-(47), which define the energy components, the relation (62) can be written as follows

$$
\begin{aligned}
& \mathcal{K}(t)-\mathcal{S}(t)-\mathcal{T}(t)= \\
& =-\frac{1}{2 t} \int_{B}\left[\varrho u_{i}(0) \dot{u}_{i}(0)+I_{i j} \varphi_{i}(0) \dot{\varphi}_{j}(0)+\varrho \kappa \sigma(0) \dot{\sigma}(0)\right] d V+ \\
& +\frac{1}{4 t} \int_{B}\left\{\varrho\left[u_{i}(0) \dot{u}_{i}(2 t)+\dot{u}_{i}(0) u_{i}(2 t)\right]+I_{i j}\left[\varphi_{i}(0) \dot{\varphi}_{j}(2 t)+\varphi_{i}(2 t) \dot{\varphi}_{j}(0)\right]+\right. \\
& +\varrho \kappa[\sigma(0) \dot{\sigma}(2 t)+\sigma(2 t) \dot{\sigma}(0)]\} d V-\frac{1}{2 t} \int_{0}^{t} \int_{B} \varrho \eta(0) \theta(s) d V d s+ \\
& +\frac{1}{4 t} \int_{0}^{t} \int_{B} \varrho \eta(0)[\theta(t-s)-\theta(t+s)] d V d s
\end{aligned}
$$

for $t>0$.

If we use the relations $(46),(47),(51),(54)$ and (60) we are led to the inequality

$$
\begin{aligned}
& \mathcal{T}(t) \leq \frac{1}{2 t}\left[\max _{\bar{B}} a(\mathbf{x})\right] \int_{0}^{t} \int_{B} \theta^{2}(s) d V d s \leq \\
& \leq \frac{1}{2 t m_{2}}\left[\max _{\bar{B}} a(\mathbf{x})\right] \int_{0}^{t} \int_{B} k_{i j} \theta,{ }_{i}(s) \theta_{j}(s) d V d s \leq \\
& \leq \frac{T_{0}}{2 t m_{2}}\left[\max _{\bar{B}} a(\mathbf{x})\right] \mathcal{E}(t)=\frac{T_{0}}{2 t m_{2}}\left[\max _{\bar{B}} a(\mathbf{x})\right] \mathcal{E}(0), t>0
\end{aligned}
$$

and if we pass to the limit for $t$ tends to infinity in the last inequality we obtain 
relation (55). In addition, using relations (13), (54) and (64) we deduce

$$
\begin{aligned}
& \int_{B}\left[\varrho \dot{u}_{i}(t) \dot{u}_{i}(t)+I_{i j} \dot{\varphi}_{i}(t) \varphi_{j}(t)\right] d V \leq 2 \mathcal{E}(0) \\
& \int_{B} \varrho \kappa \dot{\sigma}^{2}(t) d V \leq 2 \mathcal{E}(0) \\
& \int_{B} \sigma^{2}(t) d V \leq \frac{2}{\mu_{m}} \int_{B} \Psi(\mathbf{E}(t)) d V \leq \frac{2}{\mu_{m}} \mathcal{E}(0) \\
& \int_{B} \theta^{2}(t) d V \leq \frac{1}{a_{0}} \int_{B} a \theta^{2}(t) d V \leq \frac{2}{a_{0}} \mathcal{E}(0)
\end{aligned}
$$

In equality (63) we now use Schwarz's inequality and the relations (55) and (65) from which we deduce that

$$
\lim _{t \rightarrow \infty} \mathcal{K}(t)-\lim _{t \rightarrow \infty} \mathcal{S}(t)=\lim _{t \rightarrow \infty} \frac{1}{4 t} \int_{B}\left[\varrho \dot{u}_{i}(0) u_{i}(2 t)+I_{i j} \dot{\varphi}_{i}(0) \varphi_{j}(2 t)\right] d V
$$

We first approach point i) of Theorem. Since meas $\left(\partial B_{1}\right) \neq 0$, meas $\left(\partial B_{2}\right) \neq 0$ and $\left(u_{i}, \varphi_{i}\right) \in \hat{\mathbf{W}}_{1}(B)$, using relations (50), (54) and (60) we are led to

$$
\int_{B}\left[u_{i}(t) u_{i}(t)+\varphi_{i}(t) \varphi_{j}(t)\right] d V \leq \frac{1}{m_{1}} \int_{B} 2 \Psi(\mathbf{E}(t)) d V \leq \frac{2}{m_{1}} \mathcal{E}(0)
$$

therefore, by means of the Schwarz's inequality, we obtain

$$
\lim _{t \rightarrow \infty}\left\{\frac{1}{4 t} \int_{B}\left[\varrho \dot{u}_{i}(0) u_{i}(2 t)+I_{i j} \dot{\varphi}_{i}(0) \varphi_{j}(2 t)\right] d V\right\}=0
$$

If we consider the conclusion (68), then from equality (66) follows the relation (56). Relation (57) is obtained by simply combining relations (56) and (61).

We propose now to prove point ii) of the theorem. Because meas $\left(\partial B_{1}\right)=0$ and meas $\left(\partial B_{2}\right)=0$, deduce that we can use the decompositions (48) and (52) and relation (49) so that we get equality

$$
\begin{aligned}
& \frac{1}{4 t} \int_{B}\left[\varrho \dot{u}_{i}(0) u_{i}(2 t)+I_{i j} \dot{\varphi}_{i}(0) \varphi_{j}(2 t)\right] d V=\frac{1}{4 t} \int_{B}\left[\varrho \dot{u}_{i}^{*} u_{i}^{*}+I_{i j} \dot{\varphi}_{i}^{*} \varphi_{j}^{*}\right] d V+ \\
& +\frac{1}{4 t} \int_{B}\left\{\varrho\left[\dot{u}_{i}^{*}+\dot{U}_{i}^{0}\right] v_{i}(2 t)+I_{i j}\left[\dot{\varphi}_{i}^{*}+\Phi_{i}^{0}\right] \chi_{j}(2 t)\right\} d V+ \\
& +\frac{1}{2} \int_{B}\left[\varrho \dot{u}_{i}^{*} \dot{u}_{i}^{*}+I_{i j} \dot{\varphi}_{i}^{*} \dot{\varphi}_{j}^{*}\right] d V
\end{aligned}
$$


The inequality of Korn's type (50) and the inequality (13) underlying the following double inequality

$$
\int_{B}\left[v_{i}(t) v_{i}(t)+\chi_{i}(t) \chi_{i}(t)\right] d V \leq \frac{2}{m_{1}} \int_{B} \Psi(\mathbf{E}(t)) d V \leq \frac{2}{m_{1}} \mathcal{E}(0) .
$$

If we take into account the inequality (70) then equality (69) leads to

$$
\begin{aligned}
& \lim _{t \rightarrow \infty}\left\{\frac{1}{4 t} \int_{B}\left[\varrho \dot{u}_{i}(0) u_{i}(2 t)+I_{i j} \dot{\varphi}_{i}(0) \varphi_{j}(2 t)\right] d V\right\}= \\
& =\frac{1}{2} \int_{B}\left[\varrho \dot{u}_{i}^{*} \dot{u}_{i}^{*}+I_{i j} \dot{\varphi}_{i}^{*} \dot{\varphi}_{j}^{*}\right] d V
\end{aligned}
$$

Substituting the result of equation (71) in equality (66) and immediately obtain the conclusion (58). Finally, to obtain equality (59) will have to com-

bine results from relations (55), (58) and (61). Last statement ends the proof of Theorem 4.

Conclusion. At last we remark that the relations (56) and (58), restricted to the class of initial data for which $u_{i}^{*}=\varphi_{i}^{*}=0$, prove the asymptotic equipartition in mean of the kinetic and strain energies.

Acknowledgement. The publication of this paper is partially supported by the program CNCS-UEFISCDI grant PN-II-ID-WE-2012-4-169.

\section{References}

[1] M. A. Goodman and S. C. Cowin, A Continuum Theory for Granular Materials, Arch. Rational Mech. Anal., vol. 44(1972), 249-266

[2] S. C. Cowin and J. W. Nunziato, Linear Elastic Materials with Voids, J. Elasticity, vol. 131983, 125-147

[3] J. W. Nunziato and S. C. Cowin, A Nonlinear Theory of Elastic Materials with Voids, Arch. Rational Mech. Anal., vol. 72(1979), 175-201

[4] D. Iesan, A Theory of Thermoelastic Materials with Voids, Acta Mechanica, vol. 60(1986), 67-89

[5] D. Iesan, R. Quintanilla, Non-linear deformations of porous elastic solids, International Journal of Non-Linear Mechanics, 49(2013), 57-65.

[6] S. Chirita and M. Ciarletta, Time-weighted Surface Power Function Method for the Study of Spatial Behaviour in Dynamics of Continua, Eur. J. Mech. A/Solids, vol. 18(1999), 915-933 
[7] M. Marin, A partition of energy in Thermoelasticity of microstretch bodies Nonlinear Analysis: R. W. A., Vol. 11, 4(2010), 2436-2447

[8] M. Marin, Lagrange identity method for microstretch thermoelastic materials, Journal of Math. Analysis and Appl., Vol. 363, 1(2010), 275-286

[9] M. Marin, Some estimates on vibrations in thermoelasticity of dipolar bodies Journal of Vibration and Control, vol. 16, 1(2010), 33-47.

[10] R. A. Adams, Sobolev Spaces, Academic Press, New York, 1975.

[11] I. Hlavacek and J. Necas, On Inequalities of Korn's Type, Arch. Rational Mech. Anal., vol. 36(1970), 305-334

Marin MARIN,

Department of Mathematics and Computer Sciences,

Transilvania University of Brasov,

Bdul Iuliu Maniu, nr. 50, Brasov, Romania.

Email: m.marin@unitbv.ro

Olivia FLOREA,

Department of Mathematics and Computer Sciences,

Transilvania University of Brasov,

Bdul Iuliu Maniu, nr. 50, Brasov, Romania.

Email: olivia.florea@unitbv.ro 